\title{
AIAA 94-0190
}

Computation of Separated and Unsteady Flows with One- and Two-Equation Turbulence Models

J. A. Ekaterinaris

Navy-NASA Joint Institute of Aeronautics

NASA-Ames Research Center, MS 258-1

Moffett Field CA, 95035-1000

and

F. R. Menter

Eloret Institute

NASA-Ames Research Center, MS 229-1

Moffett Field CA, 95035-1000

\section{2nd Aerospace Sciences Meeting \& Exhibit January 10-13, 1994 / Reno, NV}




\title{
Computation of Separated and Unsteady Flows with One- and Two-Equation Turbulence Models
}

\author{
John A. Ekaterinaris \\ Navy-NASA Joint Institute of Aeronautics \\ NASA Ames Research Center \\ Moffett Field, CA 94035 \\ Florian R. Menter \\ Eloret Institute \\ NASA Ames Research Center \\ Moffett Field, CA 94035
}

\begin{abstract}
The ability of one- and two-equation turbulence models to predict unsteady separated flows over airfoils is evaluated. An implicit, factorized, upwindbiased numerical scheme is used for the integration of the compressible, Reynolds averaged Navier-Stokes equations. The turbulent eddy viscosity is obtained from the computed mean flowfield by integration of the turbulent field equations. The two-equation turbulence models are discretized in space with an upwind-biased, second order accurate total variation diminishing scheme. One and two-equation turbulence models are first tested for a separated airfoil flow at fixed angle of incidence. The same models are then applied to compute the unsteady flowfields about airfoils undergoing oscillatory motion at low subsonic Mach numbers. Experimental cases where the flow has been tripped at the leading edge and where natural transition was allowed to occur naturally are considered. The more recently developed field-equation turbulence models capture the physics of unsteady separated flow significantly better than the standard $k-\epsilon$ and $k-\omega$ models. However, certain differences in the hysteresis effects are obtained. For an untripped high-Reynolds-number flow, it was found necessary to take into account the leading edge transitional flow region in order to capture the correct physical mechanism that leads to dynamic stall.

$\dagger$ Research Associate Professor, Department of Aeronautics, Naval Postgraduate School Monterey California. Member AIAA.

$\dagger$ Research Scientist, Member AIAA.

Copyright (C) 1994 by the American Institute of Aeronautics and Astronautics. Inc. No copyright is asserted in the United States under Title 17, U.S. Code. The U.S. Government has a royalty-free license to exercise all rights under the copyright claimed herein for Government purposes. All other rights are reserved by the copyright owner.
\end{abstract}

\section{Introduction}

Application of Navier-Stokes methods to complex unsteady, turbulent flows is a field of continuous interest. An example of such a flow is the massively separated flow over airfoils at high incidence or in dynamic motion. For the computation of these flowfields, application of algebraic turbulence models, such as the Cebeci-Smith ${ }^{1}$ model, the Baldwin-Lomax ${ }^{2}$ model, the algebraic Renormalization Group (RNG) based model $^{3}$ or the Johnson-King ${ }^{4}$ model, becomes very complicated and often ambiguous. The source of ambiguity comes from the difficulty in defining characteristic length scales, e.g., boundary layer thickness required by these models. The standard $k-\epsilon^{5}$, and $k-\omega^{6}$ models and the field-equation. (one and twoequation) turbulence models developed over the last few years (Refs. 7, 8 and 9), do not have these ambiguities. The more recently developed models ${ }^{7,8,9}$ also seem to show promise for massively separated flowfields. These models have been extensively tested for attached and mildly separated steady flows. The ability of these models to resolve massively separated flows, such as flow over a wing at stall, a slender body at an angle of incidence, or unsteady separated flow at dynamic stall conditions, however, has not been systematically evaluated. The objective of this investigation is to test one and two-equation models for unsteady massively separated flows.

Some of the recently developed turbulence models have been tested for two dimensional unsteady separated flows. It was found that it is advantageous to use the Johnson-King model for separated flows where nonequilibrium effects are important. In Refs. 10-12 it was shown that for light dynamic stall, the Johnson-King model captures flow separation better and yields improved predictions of hysteresis effects, compared to standard algebraic models. In the present investigation, however, it was found that the experimental data ${ }^{13}$ used for validation of fully tur- 
leading edge transition, which has a significant effect on the development of the unsteady flowfield. The $k-\epsilon$ model has been compared to the Baldwin-Lomax model in Ref. 14 and 15 for dynamic motion. In a previous investigation ${ }^{16}$ an extensive evaluation of algebraic, half and one-equation models for the prediction of dynamic stall was conducted. A single model that could predict accurately the attached unsteady flow, the light- and deep-stall regime was not identified.

In Ref. 16 a central difference numerical procedure validated with experiments in Ref. 10 and 17, was used. It was found, however, that unsteady solutions obtained with this central differencing schemes were sensitive to grid spacing in the near wall region and to artificial smoothing. Therefore, here the upwind-biased numerical scheme described in detail in Ref. 18 is used. One and two-equation models are tested first for steady separated flow. The same models are then applied to compute unsteady flowfields over oscillating airfoils. The ability of one and twoequation models to predict unsteady attached flow, the light and deep stall regime is evaluated. The computed results appear to be grid independent and no artificial smoothing is used. Finally, it is shown that the leading edge transitional flow has a significant effect on the development of the unsteady flowfield about oscillating airfoils. The numerical scheme and the turbulence models are briefly described in the following sections.

\section{Numerical Implementation}

The thin-layer approximation of the compressible, Reynolds-averaged, Navier-Stokes equations for body-fitted coordinate system, $(\xi, \eta)$, is used. These equations are as follows

$$
\partial_{t} \hat{\mathbf{q}}+\partial_{\xi} \hat{\mathbf{F}}+\partial_{\eta} \hat{\mathbf{G}}=R e^{-1} \partial_{\eta} \hat{\mathbf{S}}
$$

here, $\hat{\mathrm{q}}$ is the conservative variable vector, $\hat{\mathbf{q}}=$ $[\rho, \rho u, \rho v, e]^{T}, \hat{\mathbf{F}}$ and $\hat{\mathbf{G}}$ are the inviscid flux vectors, and $\hat{S}$ represents the thin-layer approximation of the viscous terms in the normal direction. In the above equations all geometrical dimensions are normalized with the airfoil chord length $c$; the density $\rho$ is normalized with the free-stream density $\rho_{\infty}$; the Cartesian velocity components, $(u, v)$, of the physical domain are normalized with the free-stream speed of sound $a_{\infty}$; and the pressure $p$ is normalized with $p_{\infty}$.

The following upwind-biased, factorized, iterative, implicit numerical scheme is used to compute the mean flow.

$$
\begin{aligned}
& {\left[I+h_{\xi}\left(\nabla_{\xi}^{b} \tilde{A}_{i, k}^{+}+\Delta_{\xi}^{f} \tilde{A}_{i, k}^{-}\right)\right]^{p} } \\
& \times {\left[I+h_{\eta}\left(\nabla_{\eta}^{b} \tilde{B}_{i, k}^{+}+\Delta_{\eta}^{f} \tilde{B}_{i, k}^{-}\right.\right.} \\
&\left.\left.-R e^{-1} \delta_{\eta} \tilde{M}_{i, k}\right)\right]^{p} \\
& \times\left(\tilde{Q}_{i, k}^{p+1}-\tilde{Q}_{i, k}^{p}\right) \\
&=-\left[\left(\tilde{Q}_{i, k}^{p}-Q_{i, k}^{n}\right)+h_{\xi}\left(\hat{F}_{i+1 / 2, k}^{p}-\hat{F}_{i-1 / 2, k}^{p}\right)\right. \\
&+h_{\eta}\left(\hat{G}_{i, k+1 / 2}^{p}-\hat{G}_{i, k-1 / 2}^{p}\right) \\
&-\left.R e^{-1} h_{\eta}\left(\hat{S}_{i, k+1 / 2}^{p}-\hat{S}_{i, k-1 / 2}^{p}\right)\right]
\end{aligned}
$$

In this equation, $h_{\xi}=\Delta \tau / \Delta \xi$, etc., $\tilde{A}^{ \pm}=(\partial \tilde{F} / \partial \tilde{Q})$, etc., are the flux Jacobian matrices, and $\Delta, \nabla$, and $\delta$ are the forward, backward and central difference operators, respectively. The quantities $\tilde{F}_{i+1 / 2, k}, \tilde{G}_{i, k+1 / 2}$, and $\tilde{S}_{i, k+1 / 2}$ are numerical fluxes.

The inviscid fluxes $\hat{F}$ and $\hat{G}$ are evaluated using Osher's ${ }^{19}$ upwinding scheme. The numerical fluxes for a third-order accurate upwind-biased scheme are given by

$$
\begin{aligned}
\hat{F}_{i+1 / 2, k}=\tilde{F}_{i+1 / 2, k}+ & \frac{1}{6}\left[\Delta F_{i-1 / 2, k}^{+}+2 \Delta F_{1+1 / 2, k}^{+}\right] \\
& -\frac{1}{6}\left[\Delta F_{i+3 / 2, k}^{-}+2 \Delta F_{1+1 / 2, k}^{-}\right] \\
=\tilde{F}\left(Q_{i, k}, Q_{i+1, k}\right)+ & \frac{1}{6}\left[\Delta F^{+}\left(Q_{i+1, k}, Q_{i, k}\right)\right. \\
& \left.+2 \Delta F^{+}\left(Q_{i, k}, Q_{i+1, k}\right)\right] \\
& -\frac{1}{6}\left[\Delta F^{-}\left(Q_{i, k}, Q_{i+1, k}\right)\right. \\
& \left.+2 \Delta F^{-}\left(Q_{i+1, k}, Q_{i, k}\right)\right]
\end{aligned}
$$

Here, $\tilde{F}$ is the first-order accurate numerical flux for Osher's scheme ${ }^{19}$ given by

$$
\tilde{F}_{i+1 / 2, k}=\frac{1}{2}\left[F_{i, k}+F_{i+1, k}-\int_{Q_{i}}^{Q_{i+1}}\left\{F_{q}^{+}-F_{q}^{-}\right\} d Q\right]
$$

where $F_{q}=F_{q}^{+}+F_{q}^{-}, F_{q}^{ \pm}=\left(\frac{\partial F}{\partial Q}\right)^{ \pm}$, and $\Delta F^{ \pm}$are the corrections to obtain high-order accuracy. For the linearization of the left-hand side terms, the flux Jacobian matrices A, B are evaluated by the StegerWarming ${ }^{20}$ flux-vector splitting. The viscous fluxes $S_{i, k+1 / 2}$ are computed with central differences.

\section{Turbulence Models}

An attractive feature of one- and two-equation models is that they can be utilized in a more straightforward manner compared to algebraic models in both structured and unstructured flow solvers which are becoming increasingly more popular. The accuracy and the numerical robustness of these models should 
be further demonstrated. Among the most widely used one-equation models are the Baldwin-Barth ${ }^{7}$ $(B-B)$ and the Spalart-Allmaras ${ }^{8}(S-A)$ models. The first model was derived from the two-equation $k-\epsilon$ model by introducing some simplifying assumptions. The Spalart--Allmaras model was developed based on dimensional analysis and empirical criteria. An advantage of the above field-equation turbulence models compared to the algebraic and half-equation models is that they do not require evaluation of ambiguous length scales. One-equation models require numerical solution of only one partial differential equa tion; therefore, they are less computationally intensive compared to two-equation models. In this paper the standard versions of the Baldwin-Barth and Spalart-Allmaras models are used to compute steady and unsteady separated flows.

The most popular non-algebraic turbulence models are two-equation eddy-viscosity models. These models solve two transport equations, one for the turbulent kinetic energy $k$ and another one related to the turbulent length- (or time-) scale. Among the twoequation models, the $k-\epsilon$ model is the most widely used today. The original Jones and Launder $k-\epsilon$ model $^{5}$ and its variations have been very successful in a large variety of different flow situations, but it also has a number of well known shortcomings. From the standpoint of aerodynamics, the most disturbing is the lack of sensitivity to adverse pressure-gradients. Another shortcoming of the $k-\epsilon$ model is associated with the numerical stiffness of the equations when integrated through the viscous sublayer.

The $k-\omega$ model has been developed by Wilcox ${ }^{6}$ to overcome the shortcomings of the $k-\epsilon$ model. This model solves one equation for the turbulent kinetic energy $k$ and a second equation for the specific turbulent dissipation rate (or turbulence frequency) $\omega$. The $k-\omega$ model performs significantly better under mild adverse pressure-gradient conditions than the $k-\epsilon$ model. Another strong point of the model is the simplicity of its formulation in the viscous sublayer. The model does not employ damping functions and has straightforward boundary conditions. This leads to significant advantages in numerical stability. The $k-\omega$ model has been validated extensively ${ }^{6,21}$ for many flow cases with and without adverse pressure gradient. For all cases it was found to perform equally well or better than the $k-\epsilon$ model. The major shortcoming of the $k-\omega$ model is that the results of the model depend strongly on the freestream values, $\omega_{f}$, that are specified outside the shear-layer.

The free stream dependency of the original Wilcox $k-\omega$ model has, been investigated in detail in Ref. 22 , and it has been shown that the magnitude of the eddy viscosity can be changed by more than $100 \%$ just by using different values for $\omega_{f}$. This is clearly unacceptable and corrections are necessary to ensure unambiguous solutions. The standard $k-\omega$ model developed by Wilcox has been modified in Ref. 9 so that the computed solutions are insensitive to the freestream values of $\omega_{f}$. This modified model is called Baseline (BSL) $k-\omega$ model. The BSL $k-\omega$ model was further modified ${ }^{9}$ in order to improve the predictions of strong adverse pressure gradient separated flows, this model is called Shear Stress Transport (SST) $k-\omega$ model. In this paper, the SST $k-\omega$ turbulence model will be extensively tested for unsteady flows. This model has been tested in Ref. 9 for a wide class of steady separated flows and has shown good agreement with experiments.

\section{Baldwin-Barth (B-B) model}

The eddy viscosity of the Baldwin-Barth oneequation model ${ }^{7}$ is given by $\nu_{t}=\nu c_{\mu} f_{\mu} R_{T}=\nu c_{\mu} f_{3} \tilde{R}_{T}$ here $R_{T}$ is the turbulent Reynolds number and $\tilde{R}_{T}$ the modified turbulent Reynolds number. The quantity $\tilde{R}_{T}$ is the solution of the following field equation

$$
\begin{aligned}
\frac{D\left(\nu \tilde{R}_{T}\right)}{D t}= & \left(c_{\epsilon_{2}} f_{2}-c_{\epsilon_{1}}\right) \sqrt{\nu \tilde{R}_{T} P} \\
& +\left(\nu+\frac{\nu_{t}}{\sigma_{\epsilon}}\right) \nabla^{2}\left(\nu \tilde{R}_{T}\right) \\
& -\frac{1}{\sigma_{\epsilon}}\left(\nabla \nu_{t}\right) \cdot \nabla\left(\nu \tilde{R}_{T}\right)
\end{aligned}
$$

This is a partial differential equation for the field quantity $R_{T}=k^{2} / \nu \epsilon=\tilde{R}_{T} f_{3}\left(\tilde{R}_{T}\right)$, and

$$
\begin{aligned}
\frac{1}{\sigma_{\epsilon}}= & \left(c_{\epsilon_{2}}-c_{\epsilon_{1}}\right) \sqrt{c_{\mu}} / \kappa^{2} \\
\nu_{t}= & c_{\mu}\left(\nu \tilde{R}_{T}\right) D_{1} D_{2} \\
\mu_{t}= & \rho \nu_{t} \\
f_{\mu}= & D_{1} D_{2} \\
D_{1}= & 1-\exp \left(-y^{+} / A^{+}\right) \\
D_{2}= & 1-\exp \left(-y^{+} / A_{2}^{+}\right) \\
P= & \nu_{t}\left(\frac{\partial U_{i}}{\partial x_{j}}+\frac{\partial U_{j}}{\partial x_{i}}\right) \frac{\partial U_{i}}{\partial x_{j}}-\frac{2}{3} \nu_{t}\left(\frac{\partial U_{i}}{\partial x_{j}}\right) \\
f_{2}\left(y^{+}\right)= & \frac{c_{\epsilon_{1}}}{c_{\epsilon_{2}}}+\left(1-\frac{c_{\epsilon_{1}}}{c_{\epsilon_{2}}}\right)\left(\frac{1}{\kappa y^{+}}+D_{1} D_{2}\right) \\
& \left\{\sqrt{D_{1} D_{2}}+\frac{y^{+}}{\sqrt{D_{1} D_{2}}}\left(\frac{1}{A^{+}} \exp \left(-y^{+} / A^{+}\right) D_{2}\right.\right. \\
& \left.\left.+\frac{1}{A_{2}^{+}} \exp \left(-y^{+} / A_{2}^{+}\right) D_{1}\right)\right\}
\end{aligned}
$$


where $y^{+}=u_{\tau} y / \nu$ and $u_{\tau}$ is the skin friction velocity. The constants of the model are:

$$
\begin{array}{rlrl}
r & =0.41, & c_{\epsilon_{1}}=1.2, & c_{\epsilon_{2}}=2.0 \\
c_{\mu}=0.09, & A^{+}=26 . & A_{2}+=10 .
\end{array}
$$

This model is applied to the entire flowfield to compute the eddy viscosity.

\section{Spalart-Allmaras (S-A) model}

The second one-equation model used is the SpalartAllmaras (S-A) model ${ }^{8}$. The eddy viscosity is obtained from the solution of the following partial differential equation for $\tilde{\nu}$

$$
\begin{aligned}
\frac{D \tilde{\nu}}{D t}= & c_{b 1}\left(1-f_{t 2}\right) \tilde{S} \tilde{\nu} \\
& +\frac{1}{\sigma}\left[\nabla \cdot((\nu+\tilde{\nu}) \nabla \tilde{\nu})+c_{b 2}(\nabla \tilde{\nu})^{2}\right] \\
& -\left[c_{w 1} f_{w}-\frac{c_{b 1}}{\kappa^{2}} f_{t 2}\right]\left[\frac{\tilde{\nu}}{d}\right]^{2}+f_{t 1} \Delta U^{2}
\end{aligned}
$$

here $S$ is the vorticity magnitude and $\tilde{S}=S+$ $\frac{\tilde{\nu}}{\kappa^{2} d^{2}} f_{v 2}, f_{v 2}=1-\frac{\chi}{1+\chi f_{v 1}}, f_{v 1}=1-\frac{\chi^{3}}{1+\chi^{3}+c_{v 1}}, c_{v 1}=$ 7.1 and $d$ is the distance to the closest wall. The other functions of the model are:

$$
\begin{aligned}
f_{t 1} & =c_{t 1} g_{t} \exp \left(-c_{t 2} \frac{\omega_{t r}^{2}}{\Delta U^{2}}\left[d^{2}+g_{t}^{2} d_{t}^{2}\right]\right) \\
f_{t 2} & =c_{t 3} \exp \left(-c_{t 4} \chi^{2}\right) \\
g_{t} & =\min \left(0.1, \Delta U / \omega_{t r} \Delta x\right)
\end{aligned}
$$

where $\chi=\tilde{\nu} / \nu$ and $\omega_{t r}$ is used here to denote the vorticity at the wall at the boundary layer trip point. The constants of this model have been chosen the same as in the original reference ${ }^{8}$. The constants of the model are:

$$
\begin{aligned}
c_{b 1} & =0.1355, \quad c_{b 2}=0.622, \quad \sigma=2 / 3 \\
c_{w 1} & =\frac{c_{b 1}}{\kappa^{2}}+\frac{1+c_{b 2}}{\sigma}, \quad c_{w 2}=2.0, \quad c_{w 3}=0.3 \\
\kappa & =0.41, \quad f_{w}=g\left(\frac{1+c_{w 3}^{6}}{g^{6}+c_{w 3}^{6}}\right)^{1 / 6} \\
g & =r+c_{w 2}\left(r^{6}-r\right), r=\frac{\nu_{t}}{S \kappa^{2} d^{2}} \\
c_{t 1} & =1.0, \quad c_{t 2}=2.0, \quad c_{t 3}=1.1, \quad c_{t 4}=2.0
\end{aligned}
$$

The eddy viscosity is given as

$$
\nu_{t}=\tilde{\nu} f_{v 1}
$$

and the Reynolds shear stress are given by $-\overline{u_{i} u_{j}}=$ $2 \nu_{i} \sigma_{i j}$.
Original $k-\omega$ model

The original $k-\omega$ model is given by

$$
\begin{aligned}
& \frac{D \rho k}{D t}=\tau_{i j} \frac{\partial u_{i}}{\partial x_{j}}-\beta^{*} \rho \omega k+\frac{\partial}{\partial x_{j}}\left[\left(\mu+\sigma_{k}^{W} \mu_{t}\right) \frac{\partial k}{\partial x_{j}}\right] \\
& \frac{D \rho \omega}{D t}=\frac{\gamma^{W}}{\nu_{t}} \tau_{i j} \frac{\partial u_{i}}{\partial x_{j}}-\beta \rho \omega^{2}+\frac{\partial}{\partial x_{j}}\left[\left(\mu+\sigma_{\omega}^{W} \mu_{t}\right) \frac{\partial \omega}{\partial x_{j}}\right]
\end{aligned}
$$

The constants of the original Wilcox model are

$$
\begin{aligned}
\sigma_{k}^{W}=0.5, \quad \sigma_{\omega}^{W}=0.5, \quad \beta^{W}=0.0750, \\
\beta^{*}=0.09, \quad \kappa=0.41, \quad \gamma^{W}=\beta^{W} / \beta^{*}-\sigma_{\omega}^{W} \kappa^{2} / \sqrt{\beta^{*}}
\end{aligned}
$$

\section{BSL $k-\omega$ model}

The (BSL) model is identical to the $k-\omega$ model of Wilcox ${ }^{6}$ for the inner region of a boundary layer (up to approximately $\delta / 2$ ) and gradually changes to the standard $k-\epsilon$ model in the outer wake region. In order to enable computations with one set of equations, the $k-\epsilon$ model was first transformed into a $k-\omega$ formulation. The blending between the two regions is performed by a blending function that gradually changes from one to zero in the desired region. No a priori knowledge of the flowfield is necessary to perform the blending. The function also ensures that the $k-\epsilon$ formulation is selected for free shear layers. The performance of the new (BSL) model is very similar to that of the Wilcox $k-\omega$ model for adverse pressure gradient boundary-layer flows (and therefore better than that of the $k-\epsilon$ model), but without the undesirable freestream dependency. For free shear layers the new model is basically identical to the $k-\epsilon$ model, which predicts spreading rates more accurately than the $k-\omega$ model. The Baseline (BSL) $k-\omega$ model is

$$
\begin{aligned}
\frac{D \rho k}{D t} & =\tau_{i j} \frac{\partial u_{i}}{\partial x_{j}}-\beta^{*} \rho \omega k+\frac{\partial}{\partial x_{j}}\left[\left(\mu+\sigma_{k} \mu_{t}\right) \frac{\partial k}{\partial x_{j}}\right] \\
\frac{D \rho \omega}{D t} & =\frac{\gamma}{\nu_{t}} \tau_{i j} \frac{\partial u_{i}}{\partial x_{j}}-\beta \rho \omega^{2}+\frac{\partial}{\partial x_{j}}\left[\left(\mu+\sigma_{\omega} \mu_{t}\right) \frac{\partial \omega}{\partial x_{j}}\right] \\
& +2\left(1-F_{1}\right) \rho \sigma_{\omega} \frac{1}{\omega} \frac{\partial k}{\partial x_{j}} \frac{\partial \omega}{\partial x_{j}} .
\end{aligned}
$$

where the constants of the model are computed as $\phi=$ $F_{1} \phi^{W}+\left(1-F_{1}\right) \phi^{K}$ where $F_{1}$ is a blending function as defined in Ref. 9 and $\phi^{W}$ and $\phi^{K}$ the constants for the original $k-\omega$ and the $k-\epsilon$ model, respectively. The following standard values for the $k-\epsilon$ model are used,

$$
\begin{aligned}
\sigma_{k}^{K} & =1.0, \quad \sigma_{\omega}^{K}=0.856, \quad \beta^{K}=0.0828, \\
\beta^{*} & =0.09, \quad \kappa=0.41, \quad \gamma^{K}=\beta^{K} / \beta^{*}-\sigma_{\omega}^{K} \kappa^{2} / \sqrt{\beta^{*}}
\end{aligned}
$$

corresponding to the constants $C_{\epsilon 1}=1.44, C_{\epsilon 2}=1.92$ of the $k-\epsilon$ model. 
$k-\epsilon$ model

The $k-\epsilon$ model implemented here is based on the same formulation as the BSL model, except that the switch from the Wilcox model, constants $\phi^{W}$, to the $k-\epsilon$ model, constants $\phi^{K}$, takes place not in the wake region of the boundary layer but just outside the viscous sublayer. With this formulation, the Wilcox model is only used as a sublayer model and the model is referred to as a two-layer $k-\epsilon$ model.

\section{SST $k-\omega$ model}

Although the original and the new BSL $k-w$ model perform better in adverse pressure gradient flows than the $k-\epsilon$ model, they still underpredict the amount of separation for severe adverse pressure gradient flows. ${ }^{9}$ In an attempt to improve matters, the eddy-viscosity formulation of the BSL model is modified to account for the transport effects of the principal turbulent shear stress. The motivation for this modification comes from the Johnson-King ( $\mathrm{J}-$ K) model ${ }^{4}$ which has proven to be highly successful for adverse pressure gradient flows. The J-K model is based on the assumption that the turbulent shear stress is proportional to the turbulent kinetic energy in the logarithmic and wake regions of a turbulent boundary layer. Johnson and King solve an equation for the maximum turbulent shear stress at each downstream station and limit the eddy viscosity in order to satisfy this proportionality. In the framework of two-equation models, the turbulent kinetic energy is already known and it is therefore only necessary to limit the eddy viscosity to account for the same effect. The resulting model is called Shear Stress Transport (SST) model. For the SST $k-\omega$ model, the constants $\phi^{W}$ of the BSL model are replaced by the constants $\phi^{S}$ as follows:

$$
\begin{aligned}
& \sigma_{k}^{S}=0.85, \sigma_{\omega}^{S}=0.5, \quad \beta^{S}=0.0750, \quad \alpha=0.31 \\
& \beta^{*}=0.09, \quad \kappa=0.41, \quad \gamma^{S}=\beta^{S} / \beta^{*}-\sigma_{\omega}^{S} \kappa^{2} / \sqrt{\beta^{*}}
\end{aligned}
$$

where the same convention $\phi=F_{1} \phi^{S}+\left(1-F_{1}\right) \phi^{K}$ is used and the eddy viscosity is given by

$$
\nu_{t}=\frac{a_{1} k}{\max \left(\alpha \omega ; \Omega F_{2}\right)}
$$

Where $\Omega$ is the vorticity magnitude and $F_{2}=\tanh$ $\left(a r g_{2}^{2}\right)$ with $\arg _{2}=\max \left(2 \frac{\sqrt{k}}{0.09 \omega y} ; \frac{500 \nu}{y^{2} \omega}\right)$

\section{Results and Discussion}

The main objective of the present investigation is to assess the accuracy of one- and two-equation turbulence models for the computation of unsteady, massively separated, high Reynolds number airfoil flows. Experimental measurements for dynamic stall are usually available in the form of unsteady loads (lift coefficient $C_{l}$, drag coefficient $C_{d}$ and pitching moment coefficient $C_{m}$ ). Accurate computation of these quantities for unsteady flow is important, and usually is sufficient for engineering and design applications. Sometimes unsteady surface pressures are also reported.

To confirm that the turbulence models under consideration had been implemented correctly, computations were first performed for a standard incompressible airfoil test case ${ }^{23}$ with trailing edge separation. Solutions over the NACA-4412 airfoil are obtained at $\alpha=13.87^{\circ}$ angle of attack and $R e_{c}=1.52 \times 10^{6}$. In the experiment of Ref. 23 , the measurements are obtained for low speed incompressible flow. Here, the solutions with different turbulence models are obtained at $M_{\infty}=0.2$. The computed surface pressure coefficients are compared with the measurements of Ref. 23 in Fig. 1.

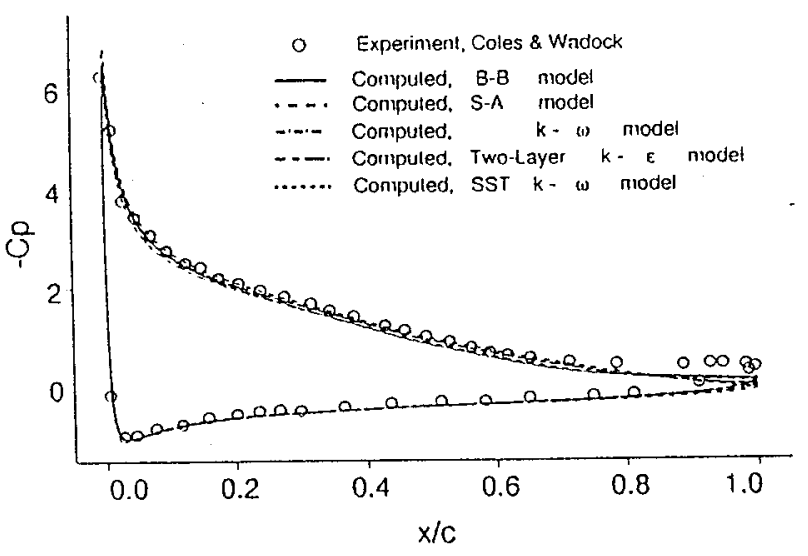

Fig. 1 Comparison of the computed and measured surface pressure coefficient with the experiment of Ref. $24 ; \alpha=13.87^{\circ}, R e=1.52 \times 10^{6}$.

Only small differences in the computed surface pressure coefficients are obtained in the trailing edge region. The computed velocity profiles at various streamwise locations are compared with the measured values in Fig. 2. 


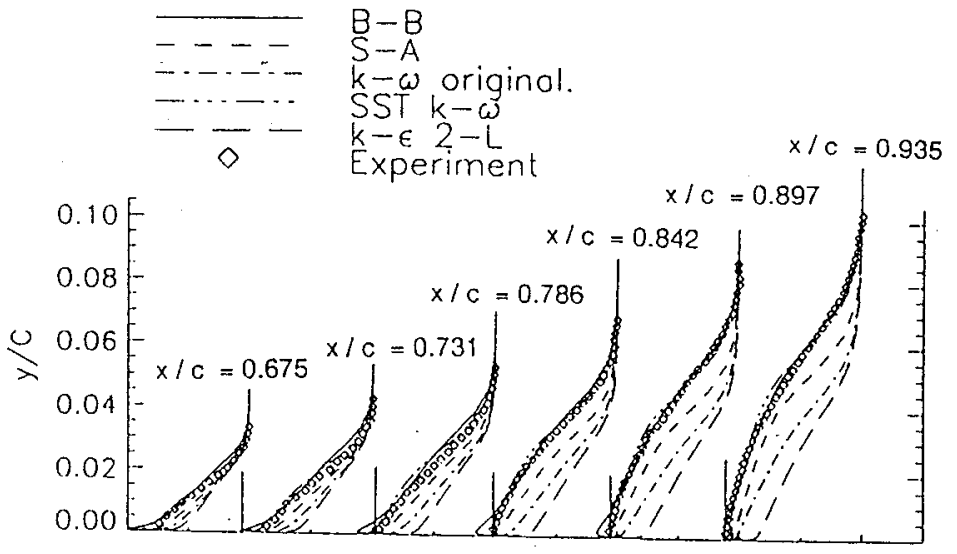

$u / U_{\delta}$

Fig. 2 Comparison of the computed and measured boundary layer profiles with the experiment of Ref. 24 for $\mathrm{x} / \mathrm{c}=0.675,0.731,0.786,0.842,0.897$, and $0.935 ; \alpha=13.87^{\circ}, \operatorname{Re}=1.52 \times 10^{6}$.

The $\mathrm{B}-\mathrm{B}$ model overpredicts separation and the $\mathrm{S}-\mathrm{A}$ model yields less separation compared to the experiment. The $k-\epsilon$ and the $k-\omega$ models predict attached flow. The SST $k-\omega$ model predicts the separation and profile shape fairly well. The trends observed for this separated flow at a fixed angle of attack carry over to the unsteady case and help to interpret the computed unsteady results.

\section{Fully Turbulent Tripped Unsteady Flows}

For validation of unsteady, fully turbulent solutions, the experimental measurements of Ref. 24 for a NACA-0015 airfoil are used, because in this experiment, as opposed to that of Ref. 13, the boundary layer was tripped at the leading edge to ensure a fully turbulent boundary layer for attached, light stall and deep stall cases. Oscillatory motions at sufficiently high angles of incidence include both massive separation during the upstroke and flow reattachment during the downstroke. The free stream Mach number is $M_{\infty}=0.3$ and the Reynolds number, based on the airfoil chord length is, $R e_{c}=2 \times 10^{6}$. In the experiment the flow was tripped at the leading edge and it is expected that the surface flow is fully turbulent. The airfoil oscillates as $\alpha(t)=\alpha_{m}+\alpha_{a} \sin (\omega t)$ with a reduced frequency $k=0.1$. The oscillation amplitude remains fixed at $\alpha_{a}=4.2^{\circ}$ and variation of the mean angle $\alpha_{m}$ leads to different flow regimes. Attached flow corresponds to $\alpha_{m}=4^{\circ}$. The light and deep stall regimes are obtained for $\alpha_{m}=11^{\circ}$, and $\alpha_{m}=15^{\circ}$, respectively.

The computations use a C-type $311 \times 91$ point grid with 130 points on the suction side and 45 points in the wake having a grid spacing $d z=0.00001$ chord lengths away from the airfoil surface as baseline grid. Normal spacing of $0.00001 c$ yields a $y^{+} \approx 2$ for the first grid point above the suction side surface. A $421 \times 151$ points grid with increased resolution in the normal to the wall separated flow region and reduced normal grid spacing of $d z=0.000005$, is also used. This grid has 111 points on the pressure side and 221 points on the suction side. Two oscillatory cycles are computed for every case and the third cycle is always identical to the second cycle. Different number of time steps for a cycle was tested, and it was found that computations with 10000,16000 and 40000 time steps gave identical solutions. The results presented are obtained using 16000 time steps, which correspond to a nondimensional time step $\Delta t \approx 0.0065$ or a Courant number $C u \approx 700$. The performance of all turbulence models is evaluated for the deep stall case. For the light stall case, solutions are computed only with the B-B, S-A and SST $k-\omega$ turbulence models, and a grid resolution study is performed. The attached unsteady flow case it is computed only with the B-B and SST $k-\omega$ models only.

$\underline{\alpha(t)=4^{\circ}+4.2^{\circ} \sin (t)}$

This flow is essentially attached and it is computed only with the B-B and SST $k-\omega$ models. Comparisons of the computed hysteresis loops with the experiment (Fig. 3) shows that the loads computed with the SST $k-\omega$ model are in good agreement with the experiment. The lift computed with the $\mathrm{B}-\mathrm{B}$ model overprealicts the experimental values and the pitching moment hysteresis does not agree with the experiment. The computed drag, however, is in agreement with the experiment. Comparison of the unsteady surface pressure coefficients (Fig. 4) at $\alpha=4^{\circ}$ and $\alpha=8^{\circ}$ during the upstroke computed by the two models, shows that the lift and pitching moment disagreements are not caused by large differences of the computed unsteady surface pressures. Due to the small trailing edge separation, the variation of the pitching moment and drag coefficients is very small (an order of magnitude less than that observed in the deep stall case). 


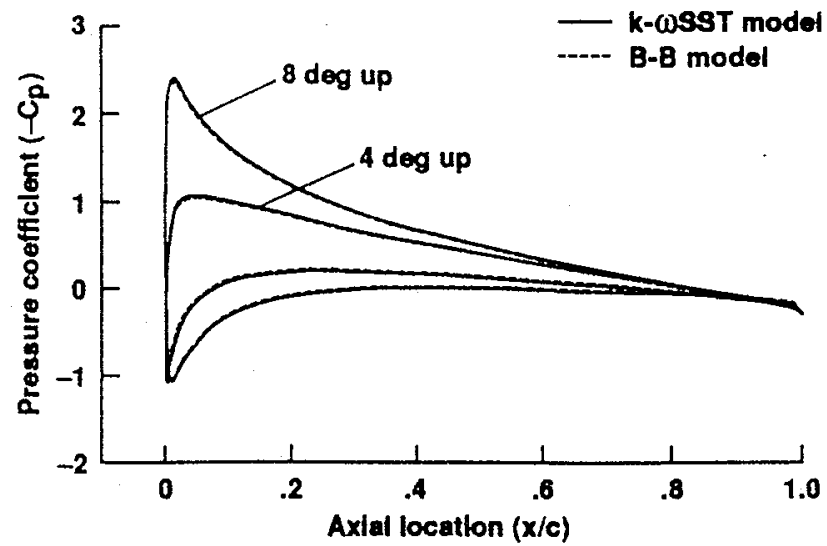

Fig. 4 Comparison of the surface pressure coefficient at $\alpha=4^{\circ}$ up and $\alpha=8^{\circ}$ up computed with the B-B and the SST $k-\omega$ models.

$\alpha(t)=11^{\circ}+4.2^{\circ} \sin (t)$

The computed solutions show that this flow is characterized by moderate trailing edge separation which develops at the peak of the cycle. The flow remains separated for a large portion of the downstroke and a recirculatory region of about half a chord length is observed. As a result, more significant hysteresis effects than the previous case are obtained. The hysteresis loops obtained from solutions using the baseline grid with the $\mathrm{B}-\mathrm{B}$, the $\mathrm{S}-\mathrm{A}$, and SST $k-\omega$ turbulence models are compared with the experimental data in Fig. 5. The B-B model predicted the most separation and yields a lower lift during reattachment but it gives good predictions for drag and pitching moment coefficients. The S-A model predicts the least separation and shows earlier flow reattachment. Similar to the S-A model, the SST $k-\omega$, model even though it shows closer agreement with the experimental lift during the initial part of the downstroke, predicted more rapid lift recovery. A solution computed on the refined $421 \times 151$ point grid with the SST $k-\omega$ turbulence model is compared with the baseline grid solution in Fig. 6. Little grid sensitivity is obtained with the refined grid.

$\alpha(t)=15^{\circ}+4.2^{\circ} \sin (t)$

The computed solution show that this unsteady flowfield is characterized by massive flow separation which develops before the peak angle of incidence.
At peak incidence and before the downstroke the dynamic stall vortex is shed and a trailing edge vortex forms. Shedding of the dynamic stall vortex causes decrease in lift and pitching moment. The flow remains separated for a large part of the downstroke and significant hysteresis effects are obtained. It is a challenge for turbulence models to be able to capture flow separation and reattachment and yield good quantitative predictions for these kind of flows. The lift, drag, and pitching moment hysteresis loops obtained from computations with the $\mathrm{B}-\mathrm{B}$ and $\mathrm{S}-\mathrm{A}$ oneequation turbulence models are compared with the experiment in Fig. 7. The lift hysteresis is predicted reasonably well by both models. The drag and pitching moment hysteresis loops indicate that both models delay onset of separation. The loads computed with the $\mathrm{B}-\mathrm{B}$ model show oscillatory behavior at the downstroke. The $\mathrm{B}-\mathrm{B}$ model predictions are in closer agreement with the experiment. A smaller extent of separated flow is obtained with the S-A model, resulting in smaller extreme values of drag and pitching moment. A more rapid flow reattachment was also observed.

Predictions of hysteresis loops obtained from solutions with the two-equation turbulence models are compared with the experiment in Fig. 8. The $k-\epsilon$, the original $k-\omega$, and the BSL $k-\omega$ (where the free stream dependency is removed) did not yield enough separation. Therefore, the loads computed with these models significantly deviated from the experimentally measured values. The solution obtained with the SST $k-\omega$ model shows large flow separation and the predictions are in close agreement with the experiment. However, at large angles of incidence and during the downstroke the loads show again oscillatory behavior.

Solutions computed with the B-B and SST $k-\omega$ models show development of a trailing edge vortex at the end of the pitch-up motion. During the downstroke this vortex convects in the wake and another trailing edge vortex forms. The second vortex initially grows in size and then convects in the wake and the vortex shedding repeats. This vortical activity and the suction side separated flowfield is shown by a series of snapshots during the downstroke in Fig. 9. In Fig. 9, the vorticity magnitude computed using the $\mathrm{B}-\mathrm{B}$ model is used to describe the flowfield. During the downstroke the trailing edge vortex shedding causes oscillations to the loads. The computed solutions for this part of the cycle are sensitive to grid distribution at the trailing edge and the wake region.

The unsteady solutions follow the trends of the steady results. The B-B model has the tendency to predict the most separation following the SST $k-\omega$ model. The S-A model tends to underpredict the 
amount of separation but not nearly to the degree of the standard $k-\omega$ and $k-\epsilon$ models. To better put the results in perspective, the pitching moment for all three oscillatory cases are plotted to the same scale in Fig. 10 and compared to the B-B and SST $k-\omega$ model solutions. The drag and pitching moment variation for the deep stall case is an order of magnitude larger compared to the variation in the attached flow and the light stall flow cases.

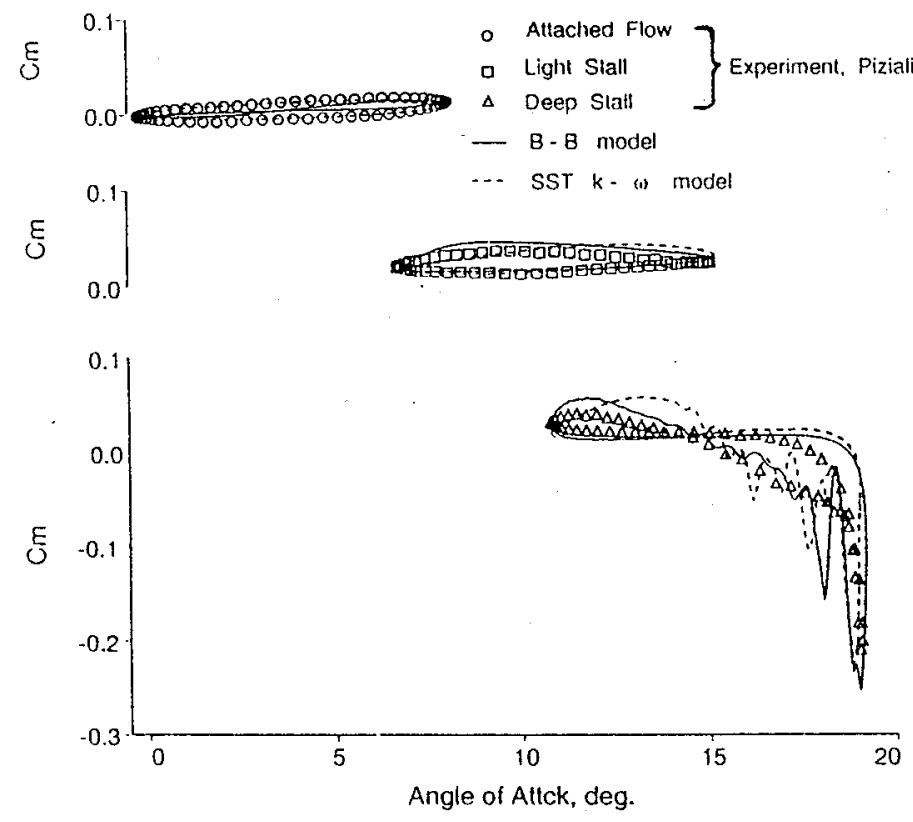

Fig. 10 Effect of increased mean angle on pitching moment.

As seen in Fig. 10, the discrepancies between experiment and computation for the attached and light stall cases are very small in relation to those for the deep stall case.

\section{The effect of transition}

A light stall case from the experimental results reported in Ref. 13 is chosen to demonstrate the effect that leading edge transition can have on the development of the unsteady flowfield. In Ref. 13, measurements in the form of integrated aerodynamic loads $\left(C_{l}, C_{d}, C_{m}\right)$ have been reported for a wide range of flow conditions and airfoil shapes. In addition, unsteady surface pressure coefficients are given. In contrast to the NACA-0015 experiment of Ref. 24, in Ref. 13 boundary layer trips were used only for limited number of deep stall cases. Unsteady solutions are obtained for a NACA-0012 airfoil executing harmonic motion $\alpha(t)=10^{\circ}+5^{\circ} \sin (\omega t)$, with a reduced frequency $k=0.1, M_{\infty}=0.3$ with untripped flow of a Reynolds number $R e_{c}=4.0 \times 10^{6}$. The same case was also considered in Refs. 10 and 11 . However, because significant hysteresis effects were not obtained for the experimental maximum angle of incidence, the amplitude of the oscillation or the mean angle were slightly increased for the computation. As a result, in both investigations a maximum angle of attack larger than $\alpha_{\max }=15^{\circ}$ reported by the experiment was reached. Experimental uncertainties and tunnel wall interference effects justified this alteration of the experimental conditions. Another reason why the maximum angle of incidence had been increased was to promote separation predicted by the turbulence models. Hysteresis effects were obtained at these larger angles of incidence, and it was concluded that massive flow separation at the trailing edge alone was responsible for stall.

Fully turbulent flow simulations of the previous section have demonstrated that the B-B model produces the most separation. The $S-A$ model produces less separation compared to the $\mathrm{B}-\mathrm{B}$ and the $\mathrm{SST}$ $k-\omega$ models. The B-B and the SST $k-\omega \bmod -$ els yield similar predictions, but the $B-B$ model is more computationally efficient. Therefore, this case is solved only with one-equation models. A solution obtained with the B-B model, which produces the most separation, for the same oscillation amplitude as the experiment ${ }^{13}$ still did not yield significant hysteresis effects. In addition, a counterclockwise loop for the pitching moment, as opposed to the clockwise loop shown in the experiment, was obtained: The same procedure of Refs. 10 and 11 was followed again, and the oscillation amplitude was "arbitrarily" increased. A solution with the B-B model was obtained with an oscillation amplitude of $5.3^{\circ}$. For the S-A model, which yields less separation, the oscillation amplitude was further increased to $5.5^{\circ}$. The loads obtained with the $\mathrm{B}-\mathrm{B}$ and the $\mathrm{S}-\mathrm{A}$ models are compared with the experiment in Fig. 11. The lift and pitching moment hysteresis reasonably agree with the experiment. The computed drag, even though it follows the experimental trends, underpredicts the extreme measured drag values. Also the phase angle where the computed drag and nose down pitching moment increases, lags the experimental values by approximately one degree. Comparison of the measured unsteady surface pressure measurements with the computed surface pressure, indicates that large discrepancies occur on the suction side, and that the agreement of the lift and the pitching moment with the experiment is coincidental.

Careful observation of the experimental surface pressure measurements shows that the lift drop, the 
increases in drag, and nose-down pitching moment is associated with a drop in the leading edge suction peak. This suction pressure drop occurs because of leading edge flow separation. The two physical mechanisms that can force the flow to separate at the leading edge are either a shock or laminar/transitional flow behavior. The progressive drop of the suction peak shown in the experiment does not support the shock separated flow assumption. Therefore, there is an indication that, even though the Reynolds number is large, the leading edge flow could be transitional. A rough approximation of the transitional flow behavior at the leading edge is performed with the following procedure. The transition onset is specified to occur immediately downstream of the suction peak location. The flow from the stagnation point until the transition onset is computed as laminar. The production term of the one-equation B-B model is set equal to zero for the laminar region. As a result, the model yields an eddy viscosity of almost zero for the laminar region. Beyond the transition point, the full production term is utilized and the computed eddy viscosity rapidly increases downstream from the transition point until a fully turbulent value is reached.

A $351 \times 91$ point grid with 170 points on the suction side is used for the numerical solution. This grid has refined resolution at the leading edge region. In the computed solutions the transitional flow region extends only over a few streamwise computational cells. A leading edge separation bubble forms at approximately 14 degrees during the upstroke and increases significantly in size before the peak of the cycle. The loads obtained from the laminar/transitional/turbulent flow solutions are compared with the experiment in Fig. 12. For comparison, the loads obtained from a fully turbulent solution are shown in the same figure. The lift hysteresis loop (Fig. 12 a) shows good qualitative agreement with the experiment. Good quantitative agreement is obtained for the upstroke but a more rapid lift recovery during part of the downstroke is observed. Similar trends are shown for the drag and pitching moment. It appears that the transitional solution predicts a more rapid flow reattachment. However, there is good agreement with the experiment for the computed nose down pitching moment and drag increase attributed to the massively separated flow during the initial part of the downstroke. It is also significant that the extreme values of the drag and pitching moment are closely predicted and the computed loads do not lag the experiment. Discrepancies obtained for the downstroke are caused by uncertainties in transition modeling and deficiencies of the turbulence miodel.
The surface pressure coefficient distributions for three angles during the upstroke, $\alpha=14.0^{\circ}, \alpha=14.5^{\circ}$ and $\alpha=14.9^{\circ}$, obtained from the fully turbulent and the transitional computations are compared with the measured values in Fig. 13. At $\alpha=14.0^{\circ}$ (Fig. 13 a) both the fully turbulent and the transitional solutions are in agreement with the experiment for the region near the leading edge. The fully turbulent solution, however, slightly overpredicts the suction peak. The surface pressure distribution obtained from the transitional solution is in close agreement with the experiment and shows the development of a leading edge separation bubble. A very small region of transitional flow is found. As the angle of attack increases to $\alpha=14.5^{\circ}$, the experiment shows a large drop in the suction peak caused by flow separation. Visualization of the computed velocity fields shows that only trailing edge separation was obtained for the fully turbulent computation. The transitional solution, on the other hand, yields more separated flow and shows formation of a vortex-like structure. At $\alpha=14.9^{\circ}$, which is the peak angle of attack of the experiment, the suction peak is further diminished and the vortical structure generated at the leading edge is convected downstream. At this angle the fully turbulent solution does not agree with the experiment and shows further increase of the suction peak. It appears that the transitional solution properly captures the physical mechanism observed in the experiment and shows development of a leading edge, vortical, dynamic-stall-like structure as the computed surface pressure coefficient demonstrates.

The values of lift and pitching moment obtained from the turbulent solution with an increased oscillation amplitude (Fig. 11) are coincidentally close to the measured values. The computed drag coefficient, however, disagrees with the experiment. Therefore, it is necessary to always compare lift, drag and pitching moment coefficients, when surface pressure measurements are not available. The fully turbulent solution predicts attached leading edge flow and trailing edge separation. The transitional solution predicts a leading edge vortex-like structure and larger overall separation, compared to the fully turbulent solution. The leading edge flow development affects significantly the suction surface flowfield and results in larger overall separation. The leading edge vortical structure is not the same as a classical dynamic stall vortex, which is clearly observed in both experiments and computations for larger oscillation amplitudes or different pitch rates. This structure forms at about 14 degrees during the upstroke and bursts in the boundary layer. 
Around the peak angle of the cycle a rapid progression of the trailing edge separation towards the leading edge is also observed.

As an approximate location for the transition onset, the maximum suction pressure point is used. For high Reynolds number fiow, the transitional region is small. It appears that in the computation, where an approximate transition onset location is determined based on the maximum suction pressure, and a simple transition model that yields an "effective" eddy viscosity for the transitional region is used, do not significantly degrade the solution for the pitch-up part of the cycle. It is not expected that these rough approximations allow accurate modeling of the complex physical mechanisms of transition, bubble formation and reattachment. It is demonstrated, however, that transition plays a significant role in the development of the unsteady separated flowfield.

\section{Conclusions}

An evaluation of the ability of one- and twoequation turbulence models in predicting hysteresis effects of unsteady fully turbulent flow over oscillating airfoils in the light and deep stall regime was conducted. None of the models considered in this investigation is capable of accurately predicting the deep stall case. However, the $B-B$, the $S-A$, and the SST $k-\omega$ models show a significant improvement over standard two-equation models. For the light stall case the S-A model did not yield sufficient separation and underpredicted the extreme values of the unsteady loads. The B-B model overpredicted the lift drop for the light stall case and the attached flow cases. The standard $k-\epsilon$ and the $k-\omega$ models did not predict separation even for the deep stall case. The SST $k-\omega$ model gave good predictions for the attached and the light stall cases.

It was found that the leading edge transitional flow is of primary importance to the overall development of the unsteady flowfield, if the flow is not tripped at the leading edge. A laminar/transitional leading-edge separation bubble developing during the pitch-up motion produces a dynamic-stall-like vortical structure. It was shown that a simple transition model significantly improves the predictions. However, accurate methods for transition modeling and prediction are still required.

Acknowledgement : The first author would like to thank Prof. M. F. Platzer, Dr. W. J. McCroskey and Dr. L. W. Carr for usefull discusions and suggestions. This investigation was supported by the Naval Warfare Center, Weapons Division, China Lake CA.

\section{References}

${ }^{1}$ Cebeci, T. and Smith, A. M., Analysis of Turbulent Boundary Layers, Academic Press, New York, 1974.

2 Baldwin, B. S. and Lomax, H., "Thin Layer Approximation and Algebraic Model for Separated Turbulent Flows," AIAA Paper 78-257, Jan. 1978.

3 Yakhot, V., and Orzag, S. A., "Renormalization Group Analysis of Turbulence. 1-Basic Theory," Journal of Scientific Computing, Vol. 1, 1986.

4 Johnson, D. A. and King, L. S., "A Mathematically Simple Turbulence Closure Model for Attached and Separated Turbulent Boundary Layers," AIAA Journal, Vol. 23, Nov. 1985, pp. 1684-1692.

5 Jones, W. P. and Launder B. E., "The Calculation of Low-Reynolds-Number-Phenomena with a Two-Equation Model of Turbulence," International Journal of Heat and Mass Transfer, Vol. 16, 1973, pp. 1119-1130.

${ }^{6}$ Wilcox, D. C., "Reassessment of the Scale-Determining Equation for Advanced Turbulence Models," AIA A Journal, Vol.26, Nov. 1988, pp.1299-1310.

${ }^{7}$ Baldwin, B. S. and Barth, T. J., "A One-Equation Turbulence Transport Model for High Reynolds Number Wall-Bounded Flows," NASA TM 102847, 1990.

${ }^{8}$ Spalart P. R. and Allmaras, S. R., "A One-Equation Turbulence Model for Aerodynamic Flows," AlAA Paper 92-0439, Jan. 1992.

${ }^{9}$ Menter, F. R., "Zonal Two-equation $k-\omega$ Turbulence Models for Aerodynamic Flows," AIAA Paper 93-2906, July 1993.

10 Clarkson, J. D., Ekaterinaris, J. A. and Platzer, M. F. "Computational Investigation of Airfoil Stall Flutter," Unsteady Aerodynamics, Aeroacoustics and Aeroelasticity of Turbomachines and Propellers, Editor H. M. Atassi, Springer-Verlag, 1991, pp. 415432 .

11 Dindar, M. and Kaynak, U., "Effect of Turbulence Modeling on Dynamic Stall of a NACA-0012 Airfoil," AIAA Paper 92-0027, Jan 1992.

12 Rumsey, C. L. and Vatsa, V. N., "A Comparison of the Predictive Capabilities of Several Turbulence Models Using Upwind and Central-Difference Computer Codes," AIAA Paper 93-0192, Jan. 1993.

13 McCroskey, W.J., "The Phenomenon of Dynamic Stall", NASA TM-81264, March 1981. 
1.t Wu, J.-C., Huff, D. L., and Sankar, L. N., "Evaluation of Three Turbulence Models in Static Air Loads and Dynamic Stall Predictions," Journal of Aircraft, Vol. 27, No. 4, April 1990, pp. 382-384.

${ }^{15}$ Rizetta, D. P. and Visbal, M. R., "Comparitive Numerical Study of Two Turbulence Models for Airfoil Static and Dynamic Stall," AIAA Journal,Vol. 31, No. 4, April 1993, pp. 784-786.

16 Srinivasan, G. R., Ekaterinaris, J. A. and McCroskey, W. J., "Dynamic Stall of an Oscillating Wing, Part 1: Evaluation of Turbulence Models," AlAA Paper 93-3403, Applied Aerodynamics Conf. Monterey, CA, Aug. 1993.

17 Jang, H. M., Ekaterinaris, J. A., Platzer, M. F., and Cebeci, T., "Essential Ingredients for the Computation of Steady and Unsteady Boundary Layers," Journal of Turbomachinery, Vol. 13, Oct. 1991, pp. 608-616.

18 Ekaterinaris, J. A., Cricelli, A. S., and Platzer, M. F., "A Zonal Method for Unsteady, Viscous, Compressible Airfoil Flows," Journal of Fluids and Structures, Vol. 8, Jan. 1994. .
${ }^{19}$ Osher, S., Solomon, F: "Upwind Difference Schemcs for Hyperbolic Systems of Conservation Laws," Mathematics of Computation, Vol. 38, No. 158, 1982, pp. $339-374$.

${ }^{20}$ Steger, J. L., Warming, R. F., "Flux Vector Splitting of the Inviscid Gas Dynamic Equations with Applications to Finite-Difference Methods," Journal of Computational Physics, Vol. 40, 1981, pp. 263-293.

21 Menter, F. R., "Performance of Popular Turbulence Models for Attached and Separated Adverse Pressure Gradient Flows," AIAA Journal, Vol. 30, Aug. 1992, pp. 2066-2072

${ }^{22}$ Menter, F. R., "Influence of Freestream Values on $k-\omega$ Turbulence Model Predictions ," AIAA Journal, Vol. 30, No. 6, 1992, pp 2066-2072.

23 Coles, D. and Wadock, A. J., "Flying-Hot-Wire Study. of Flow Past an NACA 4412 Airfoil at Maximum Lift," AIAA Journal, Vol. 17, No. 4, 1979.

24 Piziali, R. A., "An Experimental Investigation of $2 \mathrm{D}$ and $3 \mathrm{D}$ Oscillating Wing Aerodynamics for a Range of Angle of Attack Including Stall," NASA Technical Memorandum, 1993 (To be published). 

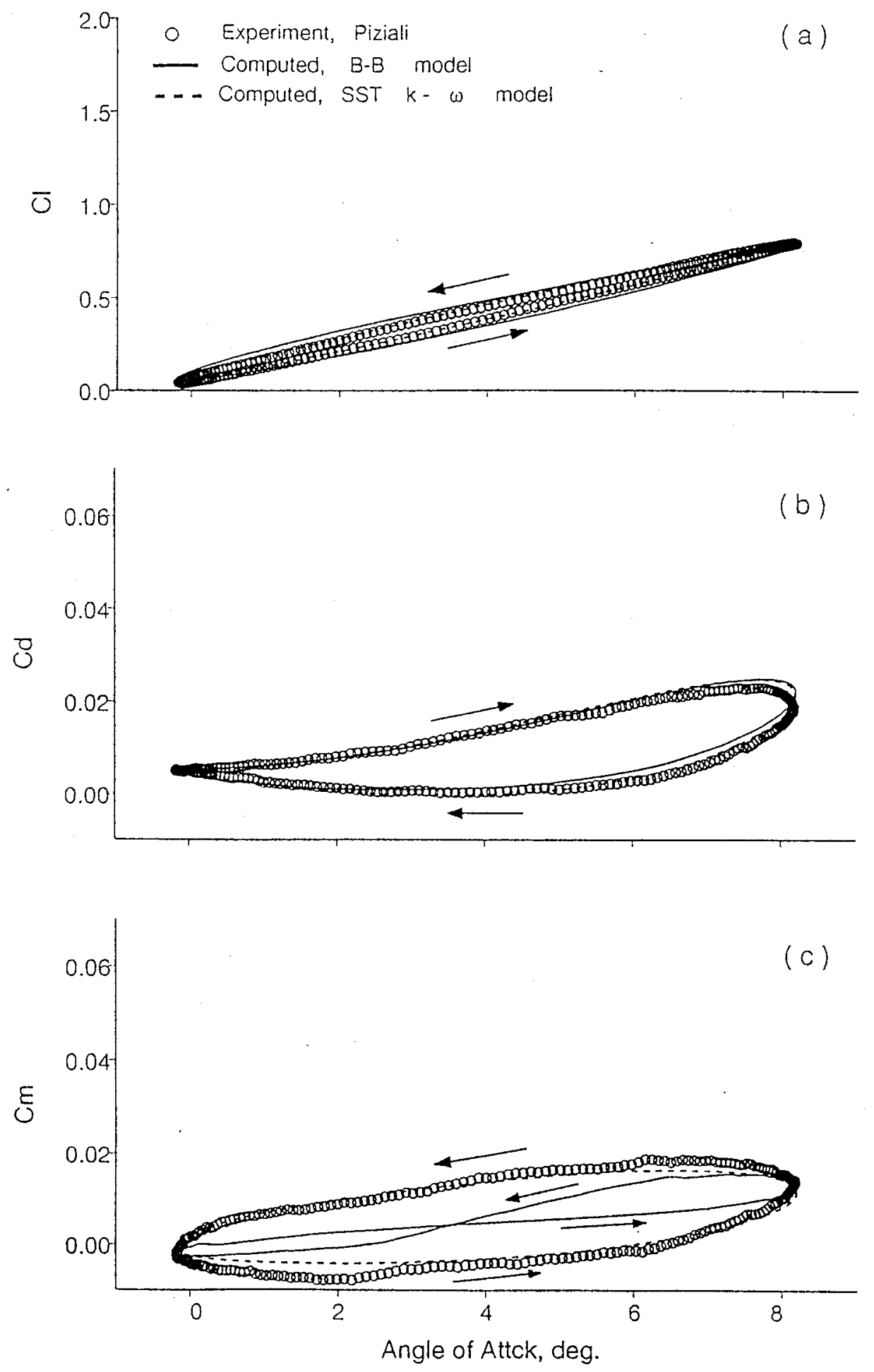

Fig. 3 Hysteresis effects for attached flow; $M=0.3, \alpha(t)=4^{\circ}+4.2^{\circ} \sin (t), k=0.1$, $R e=2 \times 10^{6}$ (fully turbulent) 

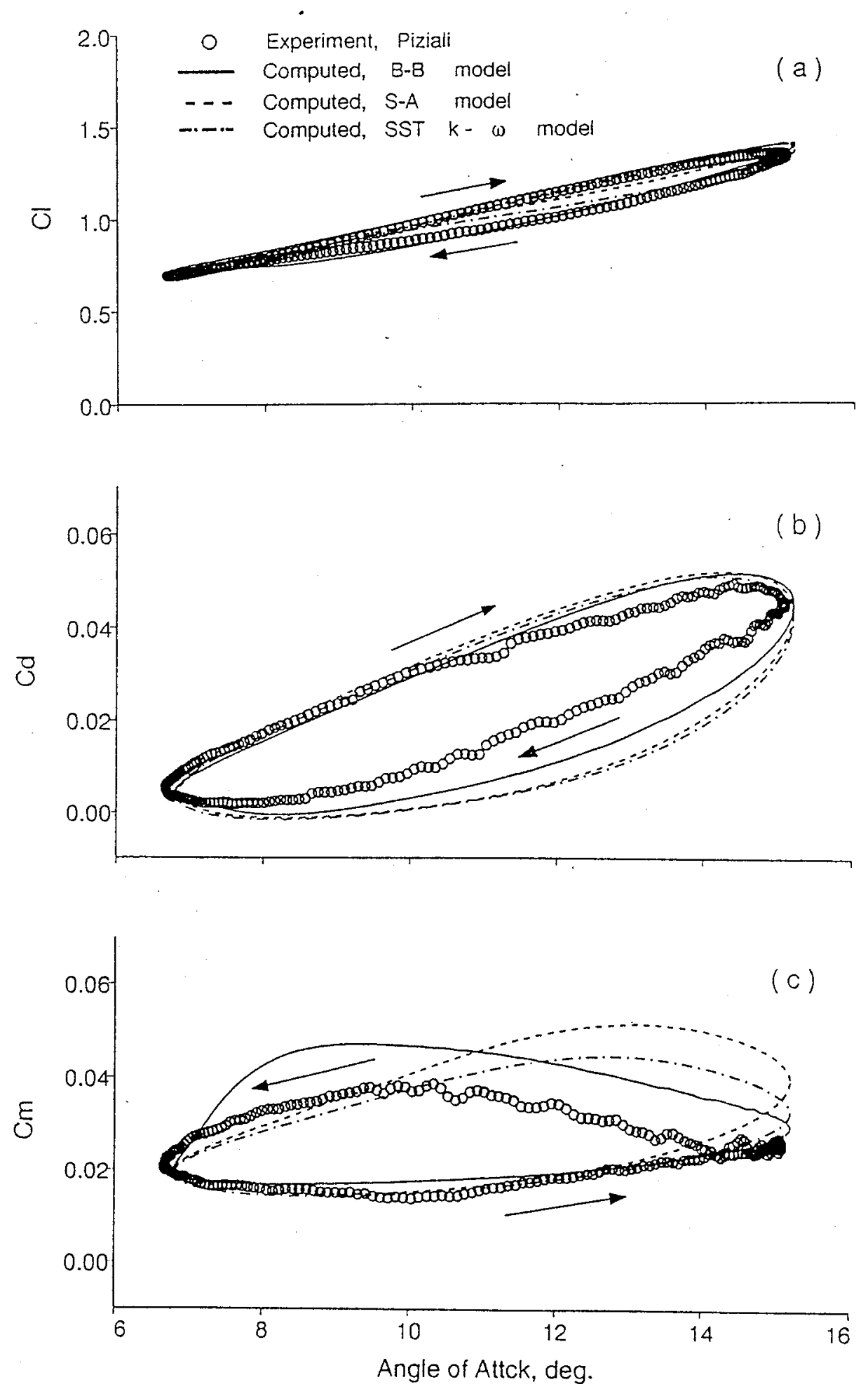

Fig. 5 Hysteresis effects for light stall flow; $M=0.3, \alpha(t)=11^{\circ}+4.2^{\circ} \sin (t), k:=0.1$, $R e=2 \times 10^{6}$ (fully turbulent). 

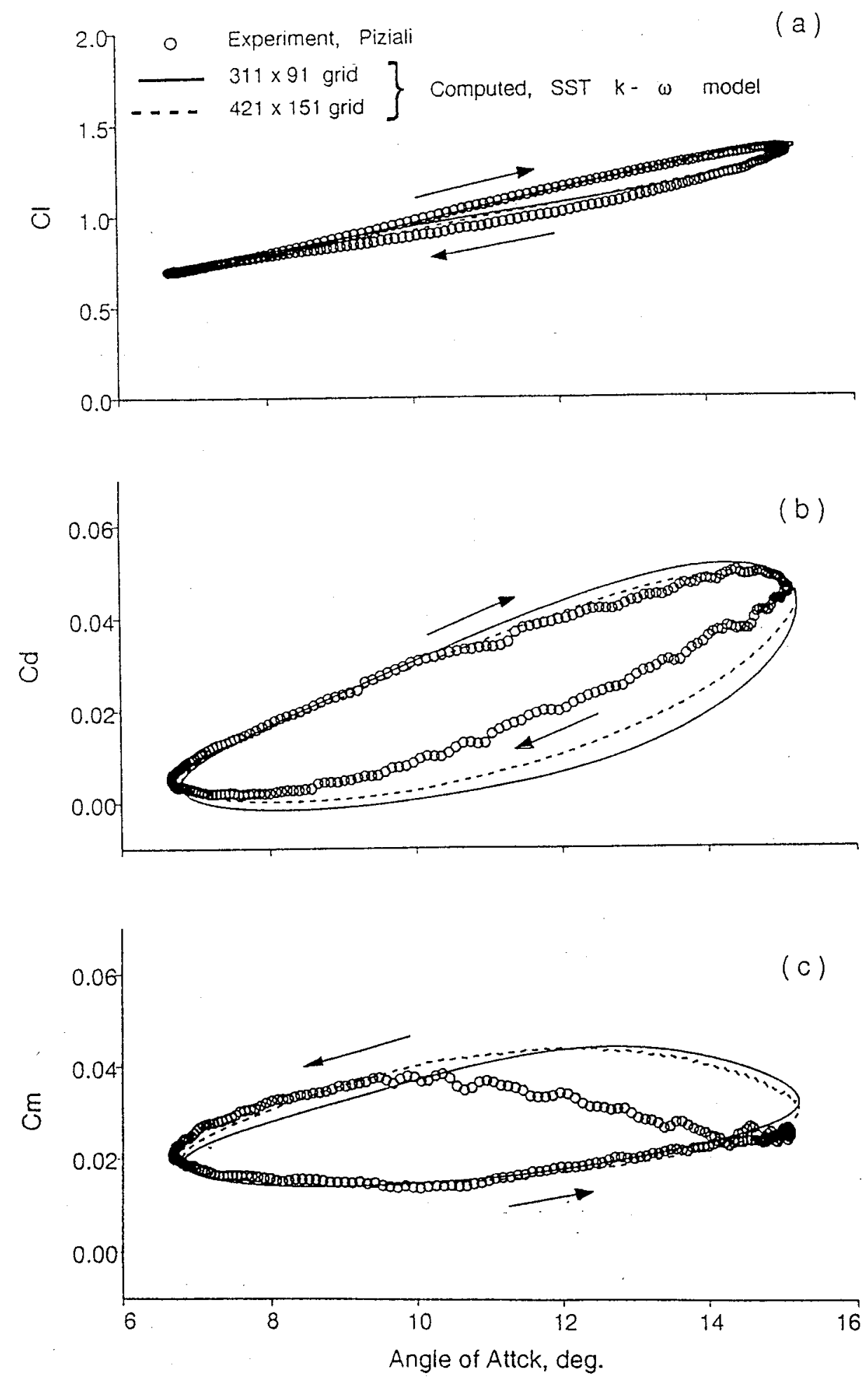

Fig. 6 Effect of grid refinement on the computed hysteresis effects; $M=0.3, \alpha(t)=11^{\circ}+4.2^{\circ} \sin (t), k=0.1, R e=2 \times 10^{6}$ (fully turbulent). 

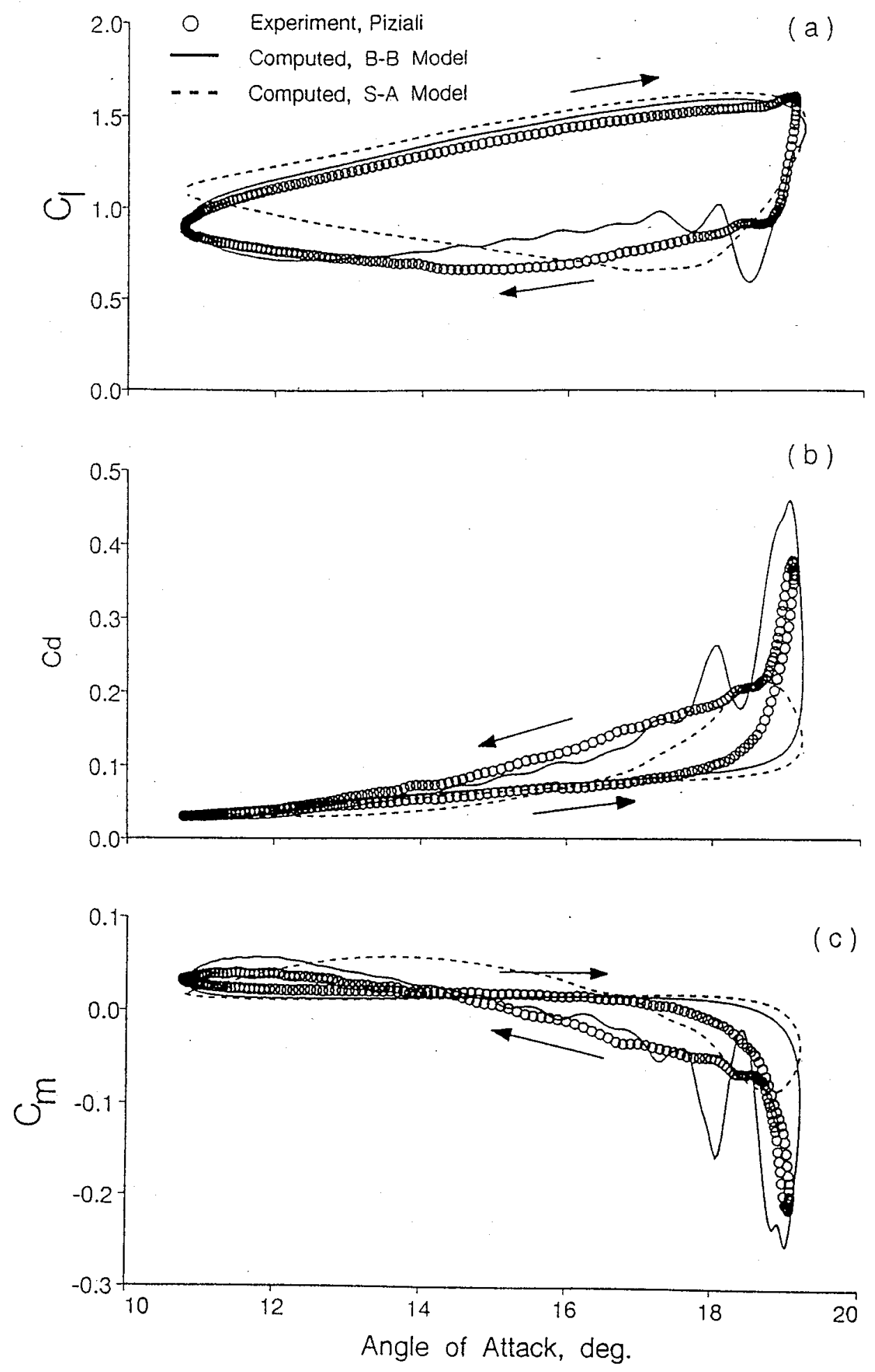

Fig. 7 Hysteresis effects for deep stall obtained with one-equation models; $M=0.3, \alpha(t)=15^{\circ}+4.2^{\circ} \sin (t), k=0.1, R e=2 \times 10^{6}$ (fully turbulent). 

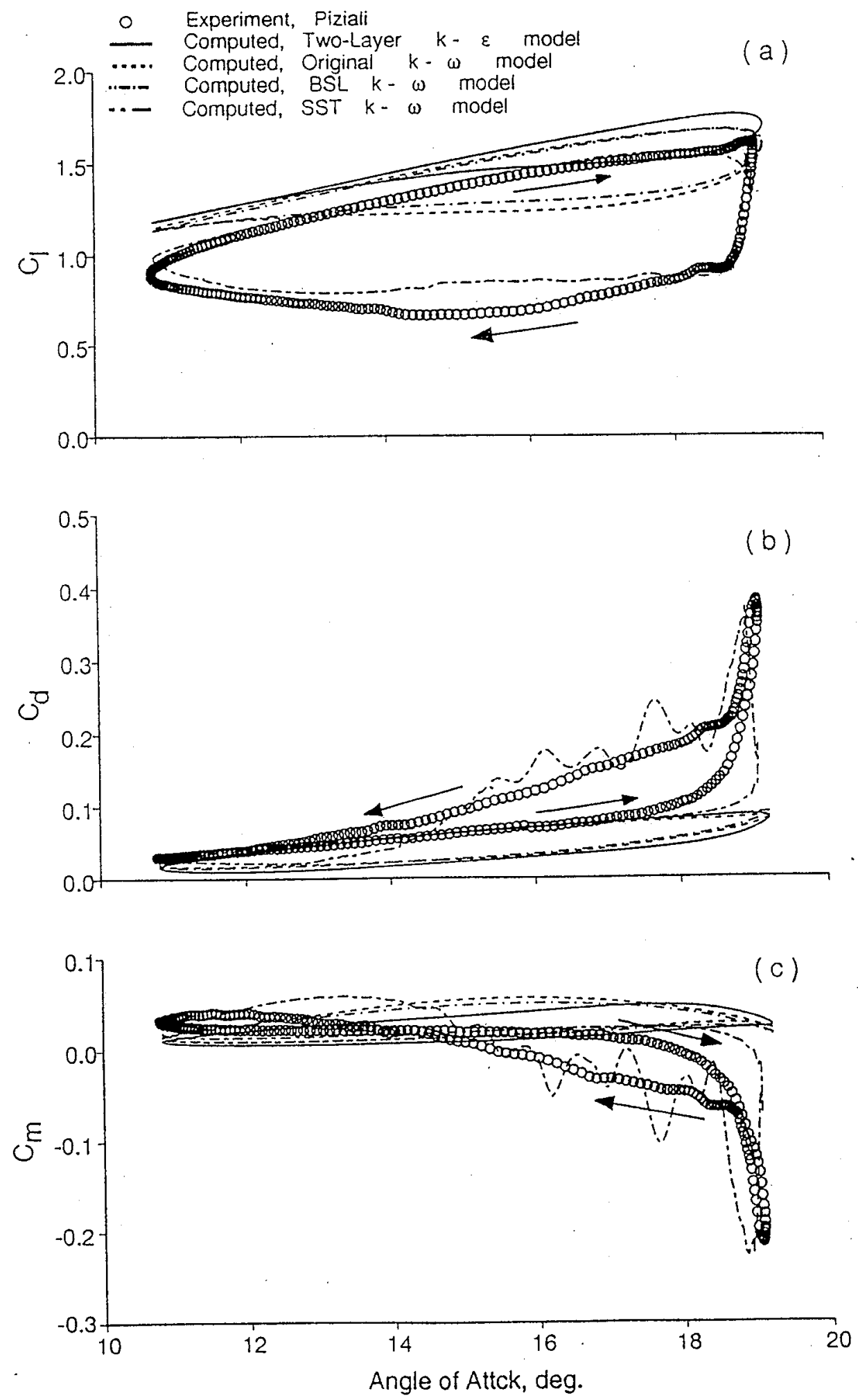

Fig. 8 Hysteresis effects for deep stall obtained with two-equation models; $M=0.3, \alpha(t)=15^{\circ}+4.2^{\circ} \sin (t), k=0.1, R e=2 \times 10^{6}$ (fully turbulent). 


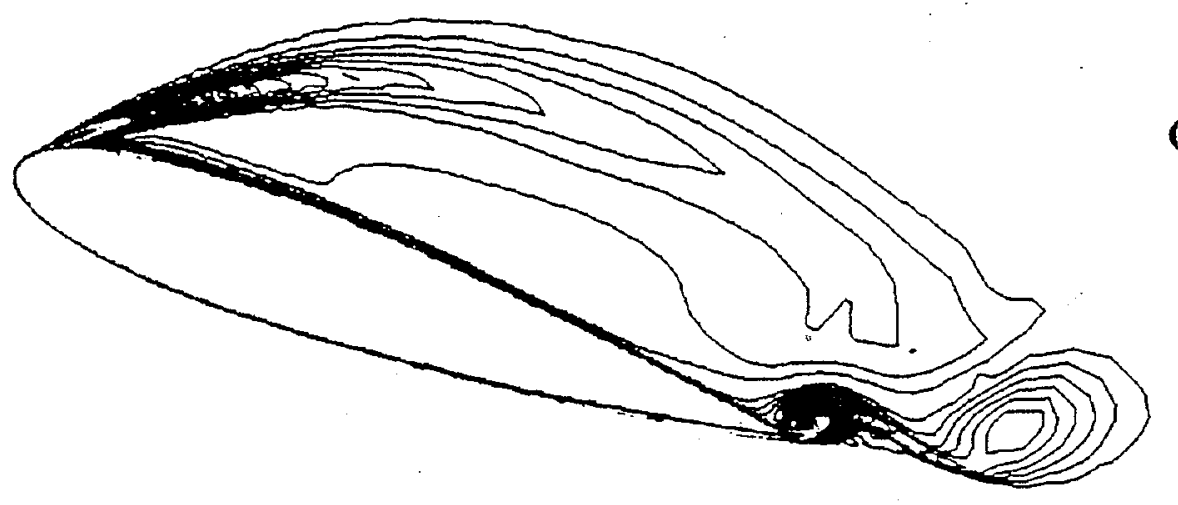

$$
\alpha=19.0^{\circ} \text { down }
$$

(a)
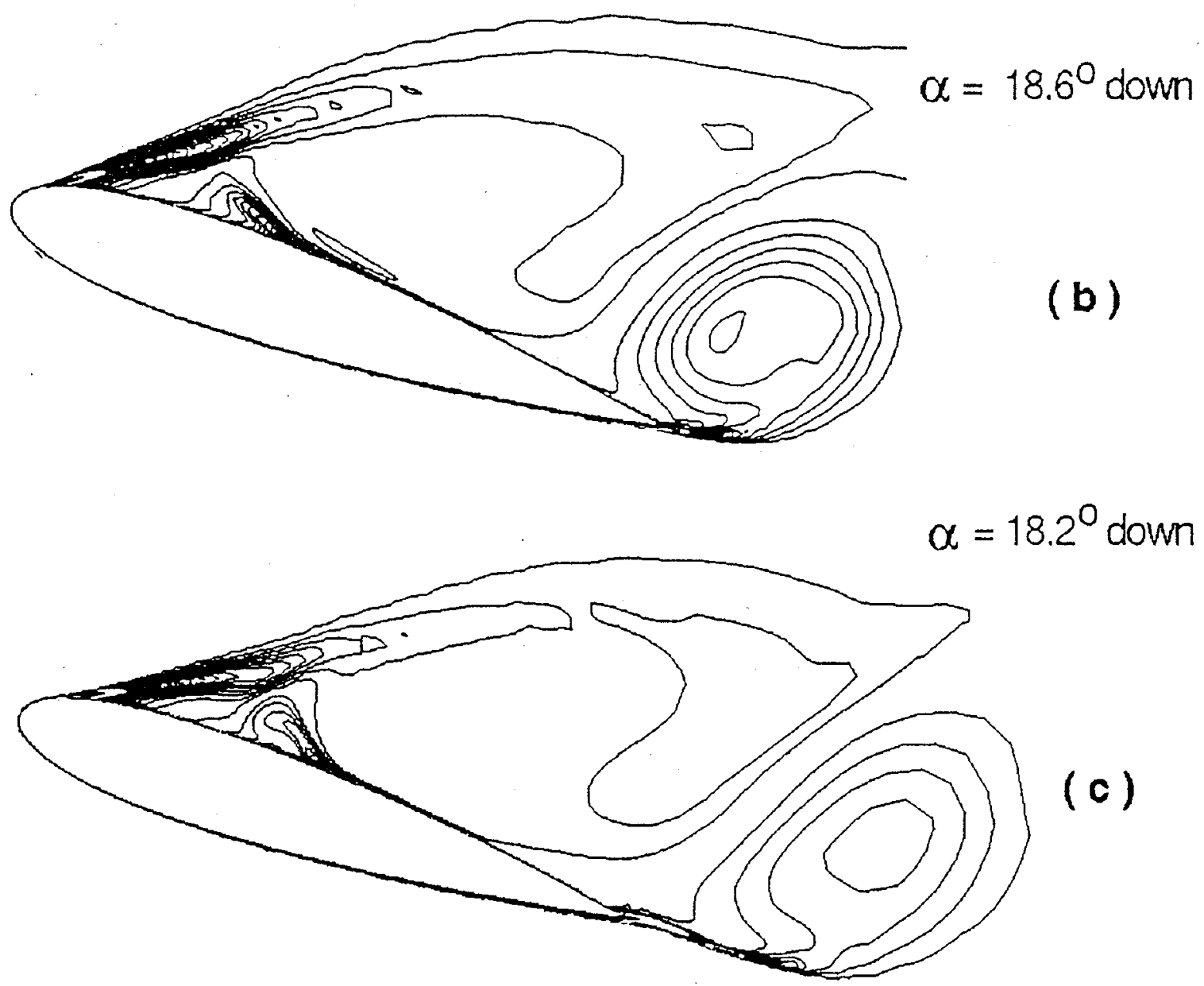

Fig. 9 Vortical flowfield development; $M=0.3, \alpha(t)=15^{\circ}+4.2^{\circ} \sin (t), k=0.1$, Re $=2 \times 10^{6}$ (fully turbulent $\mathrm{B}-\mathrm{B}$ model). 

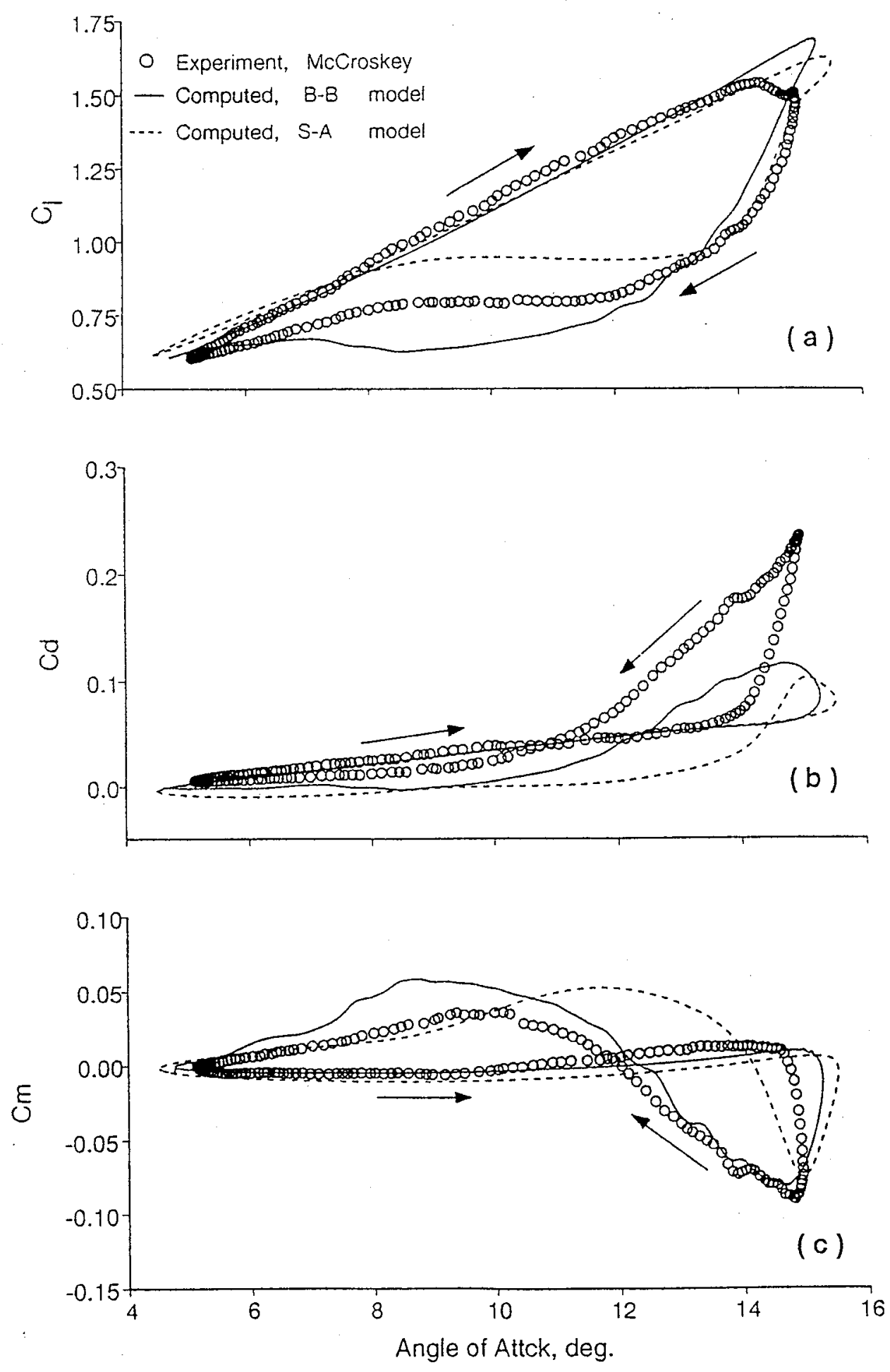

Fig. 11 Effect of increased oscillation amplitude; $M=0.3, \alpha(t)=10^{\circ}+5^{\circ} \sin (t), k=0.1$ $R e=4 \times 10^{6}$ (fully turbulent). 

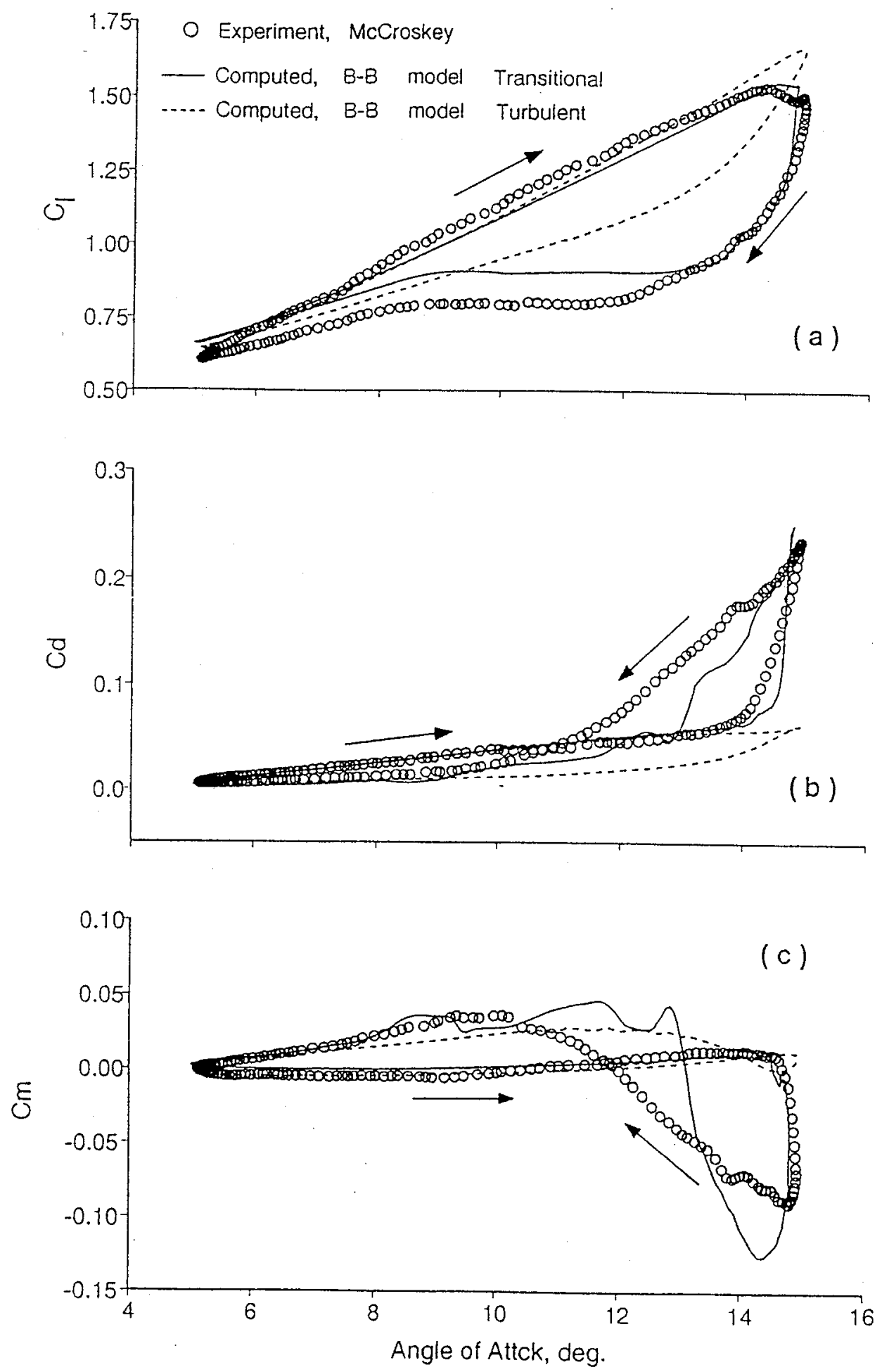

Fig. 12 Effect of leading edge transition on hysteresis effects; $M=0.3, \alpha(t)=10^{\circ}+5^{\circ} \sin (t)$, $k=0.1, R e=4 \times 10^{6}$ 

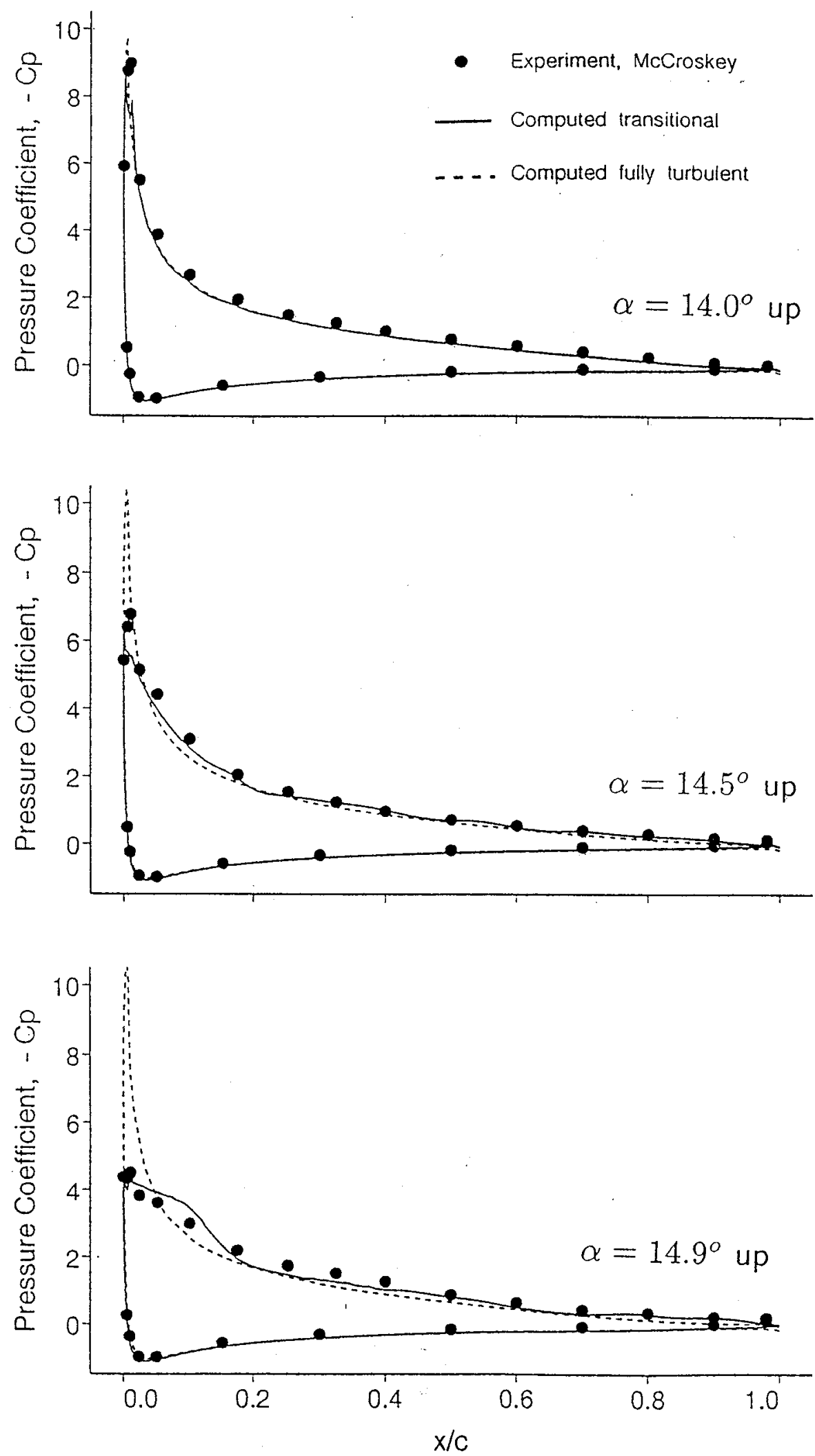

Fig. 13 Effect of transition on the computed surface pressure coefficient; $M=0.3, \alpha(t)=10^{\circ}+5^{\circ} \sin (t), k=0.1, R e=4 \times 10^{6}$ 